\title{
The thoracic spine sign in bedside ultrasound: three cases
}

\author{
Abdul-Aziz R. Ahmed ${ }^{1}$, Jennifer A. Martin², Turandot Saul', Resa E. Lewiss ${ }^{1}$ \\ ${ }^{1}$ Department of Emergency Medicine, Mount Sinai St. Luke's Hospital, Mount Sinai Roosevelt Hospital, New York, \\ NY, ${ }^{2}$ Department of Emergency Medicine, St. Francis Hospital and Medical Center, Hartford, CT, USA
}

\begin{abstract}
The "thoracic spine sign" is visualized when anechoic or hypoechoic fluid is present in the pleural space. Fluid serves as a medium through which the thoracic vertebral bodies are visualized above the diaphragm. We present three cases of emergency department patients with a thoracic spine sign identified on bedside ultrasound. These patients were subsequently diagnosed with pleural fluid accumulations on chest radiograph. Our findings suggest that the identification of the sonographic spine sign may aid the physician in the evaluation and diagnosis of emergency department patients with thoracic complaints.
\end{abstract}

Keywords: thoracic ultrasound, bedside ultrasound, thoracic spine sign, pleural effusion

\section{Introduction}

Bedside ultrasound can be an invaluable diagnostic modality in patients with thoracic pathology in the emergency department (ED) [1]. In the upper abdomen, the homogenous, blood filled liver and spleen act as excellent acoustic windows to visualize deeper structures, such as the outline of the vertebral bodies with posterior acoustic shadowing. Cephalad to these organs, the muscular diaphragm is seen as a hyperechoic stripe, moving with normal respirations. Cephalad to the diaphragm, at the lung bases, the air-filled lung parenchyma scatters ultrasound waves obscuring visualization of underlying structures.

A mirror image artifact of the liver or spleen can often be seen at the lung base. Mirroring is produced when an object is located in front of a strong reflector such as the

Received 8.03.2014 Accepted 8.04.2014

Med Ultrason

2014, Vol. 16, No 2, 179-181

Corresponding author: Turandot Saul, MD

Department of Emergency Medicine

Mount Sinai St. Luke's Hospital

Mount Sinai Roosevelt Hospital

1111 Amsterdam Avenue

New York, NY, 10025, USA 212-523-3981

E-mail: turan@joshsaul.com diaphragm. A second representation of the object is seen above the diaphragm. In the presence of pleural fluid, this mirroring is lost and an anechoic or hypoechoic area is seen. Ultrasound waves transmit well through fluid, and consquently the vertebral bodies. The "thoracic spine sign" is indicative of fluid in the pleural space [2]. We present three cases of the thoracic spine sign seen in ED patients who were all ultimately diagnosed with pleural fluid accumulation.

\section{Case 1}

A 71-year-old woman with a past medical history of diabetes, chronic renal failure, and hypertension presented to the ED with 1 month of progressively worsening shortness of breath. Physical examination was notable for decreased left sided breath sounds and $2+$ bilateral lower extremity edema. The patient was not compliant with her medications. Laboratory analysis was significant for a b-type natriuretic peptide level of $1000 \mathrm{pg} / \mathrm{ml}$ and a serum creatinine of $6.1 \mathrm{mg} / \mathrm{dl}$. A bedside thoracic ultrasound was performed (fig 1) and a left sided pleural effusion was noted with visualization of the thoracic vertebrae at the left lung base. A chest radiograph was performed and demonstrated a left pleural effusion. The patient was treated with furosemide due to volume overload and admitted to the medicine floor. 


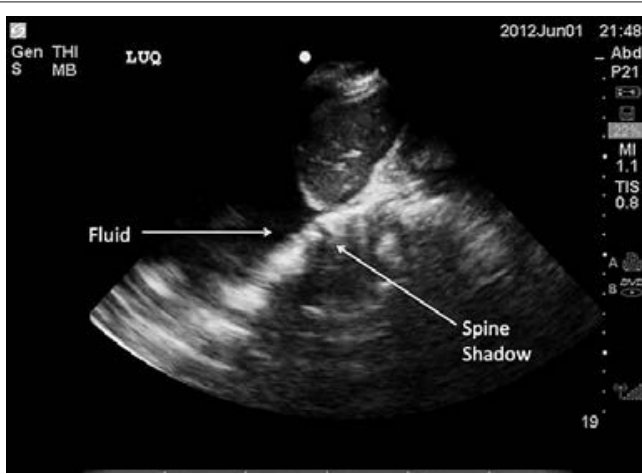

Fig 1. Coronal view of the thorax in the left midaxillary line. Pleural fluid is visualized with the thoracic spine sign.

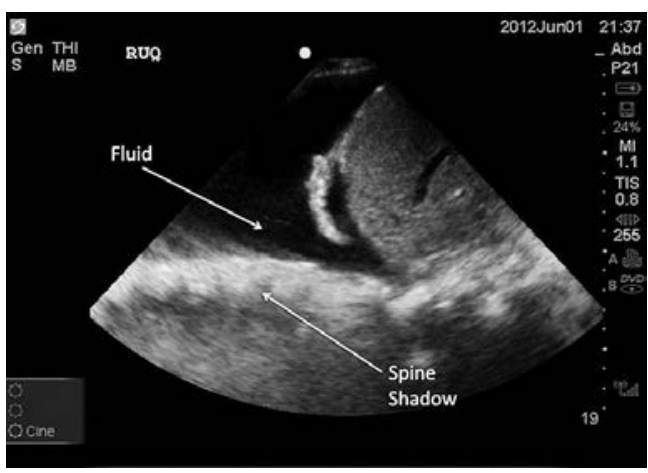

Fig 2. Coronal view of the right lung base. A large anechoic pleural effusion with visualization of vertebral bodies above the diaphragm.

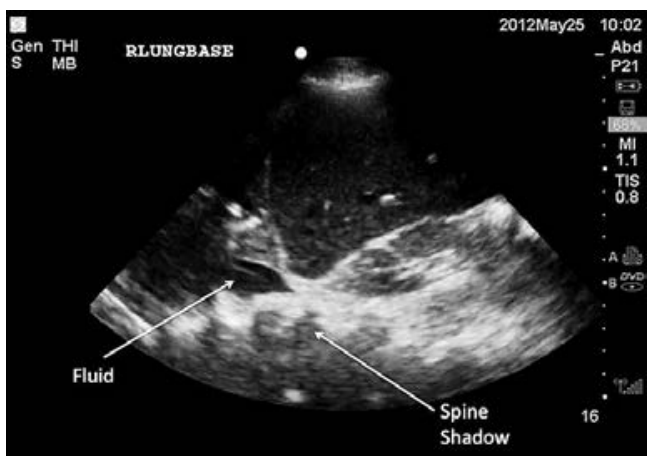

Fig 3. Coronal view of the thorax in the right midaxillary line. An area of variable echogenicity is visualized in the right hemi-thorax. Pleural fluid and the thoracic spine sign are both seen.

\section{Case 2}

A 96-year-old woman with a past medical history of hypertension was sent to the ED by her nursing home due to altered mental status and fever. Vital signs included a systolic blood pressure of $132 \mathrm{mmHg}$, heart rate of 140 beats per minute, respiratory rate of 42 breaths per minute and oxygen saturation of $96 \%$ on non re-breather facemask. The patient was unable to provide any history. Lung examination revealed decreased breath sounds with dullness to percussion at the right lung base. A bedside thoracic ultrasound was performed and a right-sided pleural effusion was noted with visualization of the thoracic vertebral bodies (fig 2). A chest radiograph confirmed the presence of a right-sided pleural effusion.

\section{Case 3}

A 26-year-old-man with no contributory past medical history presented to the ED after an altercation with two stab wounds to the right lower chest and one to the right lower quadrant of the abdomen. In the trauma resuscitation room, the patient had a blood pressure of $109 / 84 \mathrm{mmHg}$, heart rate 97 beats per minute, respiratory rate 22 breaths per minute and oxygen saturation $99 \%$ on room air. On auscultation, the physician noted equal breath sounds. The physician performed a bedside Extended Focused Assessment with Sonography for Trauma (EFAST) examination and interpreted anechoic fluid in Morison's pouch, with clear visualization of the abdominal vertebral bodies. Above the right diaphragm, there was variable echogenicity. Since the vertebral bodies continued to be visualized into the thoracic cavity (fig 3) fluid was suspected. Portable chest radiograph showed a large right hemothorax with leftward deviation of the trachea. An emergent chest tube was placed, drained $650 \mathrm{ml}$ of blood and the patient was admitted to the surgical intensive care unit.

\section{Discussion}

On bedside ultrasound, the thoracic spine cannot normally be visualized above the diaphragm because the air-filled lungs scatter the ultrasound waves. In a patient with pleural fluid, there is continued visualization of the vertebral bodies from the abdomen into the thoracic cavity.

A variety of disease processes may result in pleural fluid, including traumatic injury, congestive heart failure, pneumonia, malignancy, renal failure, cirrhosis, and pulmonary embolism. Ultrasound is known to be more sensitive than chest radiograph in the diagnosis of small pleural effusions [1,3] and is usually obvious by the loss of mirror image artifact and visualization of an anechoic area above the diaphragm. In cases where the echogenicity of the pleural fluid is 
variable (complicated effusion, empyema or clotted blood), the thoracic spine sign can aid the bedside sonographer in confirming the presence of pleural fluid.

\section{Conclusion}

Bedside ultrasound can be incorporated into a patient's initial assessment for thoracic complaints. In the three cases presented, the thoracic spine sign was seen confirming the presence of pleural fluid.

\section{References}

1. Koeze J, Nijsten MW, Lansink AO, Droogh JM, Ismael F. Bedside lung ultrasound in the critically ill patient with pulmonary pathology: differential diagnoses with comparable chest X-Ray opacification. Crit Ultrasound J 2012; 4: 1.

2. Nobel V, Liteplo A. Lung. In: Dawson M, Mallon, M (eds). Introduction to Bedside Ultrasound, Volume 1. Emergency Ultrasound Solutions 2013; 76-77.

3. Grimberg A, Shigueoka DC, Atallah AN, Ajzen S, Iared W. Diagnostic accuracy of sonography for pleural effusion: systematic review. Sao Paulo Med J 2010; 128: 90-95. 